\title{
A RARE AND FATAL COMPLICATION OF A SELF- LIMITING INFECTION: A CASE REPORT ON DENGUE ASSOCIATED HEMOPHAGOCYTIC LYMPHOHISTIOCYTOSIS
}

\author{
ALVIN OLIVER PAYUS ${ }^{1}$, CHEONG LEI WAH ${ }^{2}$, SYAHRUL SAZLIYANA SHAHARIR ${ }^{3}$
}

\begin{abstract}
Hemophagocytic lymphohistiocytosis $(H L H)$ is a life-threatening medical condition characterized by hyperphagocytosis secondary to an inappropriate over-activation of macrophages and lymphocytes that driven by excessive cytokines production which resulted in cellular destructions. It can arise de novo as a result of an autosomal recessive genetic disorder, or in the background of an infection, malignancy or autoimmune disease. Dengue fever is one of the uncommon causes of infection related secondary HLH. Here, we present a case of a Dengue associated HLH which was successfully treated with intravenous methylprednisolone and immunoglobulin $G$. In conclusion, the purpose of this case report is to illustrate the importance of early recognition and prompt initiation of the appropriate treatment for HLH suspected patient whom otherwise has high mortality rate.
\end{abstract}

Keyword: Hemophagocytic lymphohistiocytosis; cytokines; Dengue; methylprednisolone; intravenous immunoglobulin $G$.

Received: 11 January 2019

Accepted: 11 April 2019

DOI: https://doi.org/10.3329/bjmed.v30i2.41536

\section{Introduction}

Majority of Dengue infection runs on a benign and self-limiting course. Only a small proportion of cases will develop complications of bleeding diathesis and plasma leakage. Hemophagocytosis is another fatal complication that potentially occur in the background of Dengue infection which tend to be missed or delayed and has high mortality rate. We report a patient with dengue infection who was suspected to have hemophagocytic lymphohistiocytosis and successfully treated with combination of high dose corticosteroids and intravenous immunoglobulin G. To the best of our knowledge, there is very few of such cases reported. And this rare complication of Dengue infection is the centre of discussion in this case report.

\section{Case Report}

An 89-year old lady with underlying hyperlipidaemia on regular medication was presented with history of fever and feeling unwell for the past few days. She has also been having earache and dizziness, but otherwise has no respiratory nor urinary symptoms, no altered bowel habit, no vomiting and no abdominal pain. Upon arrival, she was fully alert, not tachypnoeic and was febrile with the temperature of $39.5^{\circ} \mathrm{C}$. Her pulse rate and blood pressure were normal. There was reduce breath sound with coarse crackles over the right lower zone, but there was no wheezing heard. Cardiovascular examination was normal. Her abdomen was soft, not tender and no palpable mass nor organomegaly. Her neurological examination was unremarkable. Initial laboratory investigation was taken and revealed normal haemoglobin of $11.0 \mathrm{gm} / \mathrm{dl}$, leukopenia with the white cell count of $3.8 \times 10^{9} / \mathrm{L}$, and thrombocytopenia with the platelet of $88 \times 10^{9} / \mathrm{L}$. She also has transaminitis and hyperbilirubinemia (alanine aminotransferase 436 $\mathrm{U} / \mathrm{L}$, aspartate transaminase $329 \mathrm{U} / \mathrm{L}$, and total bilirubin $60.8 \mu \mathrm{mol} / \mathrm{L}$ ), acute kidney injury (urea 23 $\mathrm{mmol} / \mathrm{L}$, creatinine $459 \mathrm{umol} / \mathrm{L}$ ) and significant coagulopathy. There were no electrolytes imbalances (sodium $142 \mathrm{mmol} / \mathrm{L}$, potassium $4.8 \mathrm{mmol} / \mathrm{L}$,

1. Medicine Based Department, Faculty of Medicine and Health Science, Universiti Malaysia Sabah, Jalan UMS, 88400 Kota Kinabalu, Sabah

2. Department of Pathology, Universiti Kebangsaan Malaysia Medical Centre (UKMMC), Jalan Yaacob Latif, 56000 Cheras, Kuala Lumpur, Malaysia

3. Department of Medicine, Universiti Kebangsaan Malaysia Medical Centre (UKMMC), Jalan Yaacob Latif, 56000 Cheras, Kuala Lumpur, Malaysia

Address of Correspondence: Dr. Alvin Oliver Payus, Senior Lecturer (Medicine), Medicine Based Department, Faculty of Medicine and Health Science, Universiti Malaysia Sabah, Jalan UMS, 88400 Kota, Kinabalu, Sabah, Email: dralvinpayus@ums.edu.my, Contact number: +6018-8703503 (hp), +6088-320 000 (fax) 
corrected calcium $2.21 \mathrm{mmol} / \mathrm{L}$, magnesium 1.04 $\mathrm{mmol} / \mathrm{L})$. In view of thrombocytopenia, leukopenia, and endemicity of the virus, Dengue infection was suspected and later confirmed by serology test which was positive for Dengue IgM. She was then given fluid replacement with $0.9 \%$ saline at rate of $1 \mathrm{~mL} / \mathrm{kg} /$ hour. Chest radiographic imaging showed consolidative changes over the right lower zone (as shown in Figure 1), which corresponded to the clinical findings. Therefore, intravenous (IV) Ceftriaxone 2gm once daily was started in order to cover for superimposed bacterial pneumonia.

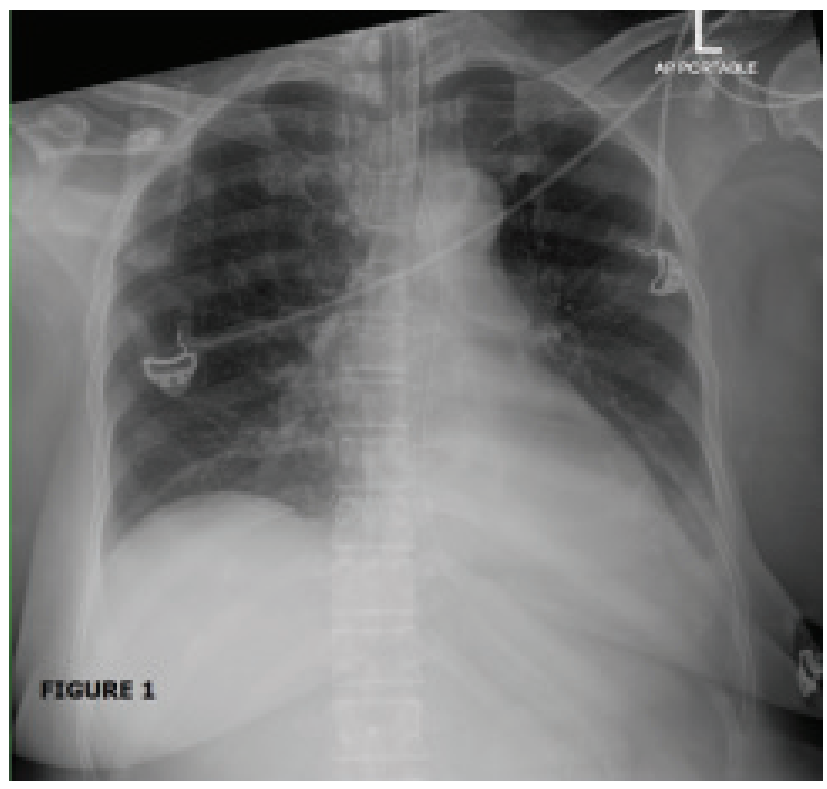

Figure 1. Chest radiograph imaging done on arrival showed ill-defined air space opacities over the left lower zones, with increased bilateral perihilar opacities. There was an apparent cardiomegaly as well. Otherwise, no pleural effusion.

Unfortunately, she desaturated few hours after admission and requires non-invasive ventilatory support. Ultrasound imaging of the abdomen showed no sonographic evidence of liver abscess or biliary duct dilatation, no splenomegaly with span of $8.1 \mathrm{~cm}$ and normal pancreas. Despite three days on IV antibiotic and supportive treatment, she was still febrile and did not show any signs of improvement. Serial blood investigations showed pancytopenia, worsening kidney injury, hepatitis and coagulopathy with hypofibrinogenemia. There was haemolysis as evidence by raised indirect bilirubin (297 umol/L), lactate dehydrogenase $(2349 \mathrm{u} / \mathrm{L})$ and transferrin saturation level (66\%). This prompted the investigation for Hemophagocytic Lymphohistiocytosis (HLH). Serum ferritin and fasting triglycerides level were taken and showed 10,382 ug/L and $12.10 \mathrm{mmol} / \mathrm{L}$ respectively, which were both markedly raised. In view of high suspicion index of HLH, she was planned for bone marrow aspiration biopsy. While waiting for the procedure to be done, IV methylprednisolone 50mg was immediately started prior to the procedure in view of high possibility of HLH, which was then continued once daily. Platelet pack was transfused prior and during the bone marrow aspiration procedure. The histopathological examination of the bone marrow biopsy showed evidence of increase histiocytes and hyperphagocytic activity, in keeping with HLH findings (as shown in Figure 2).

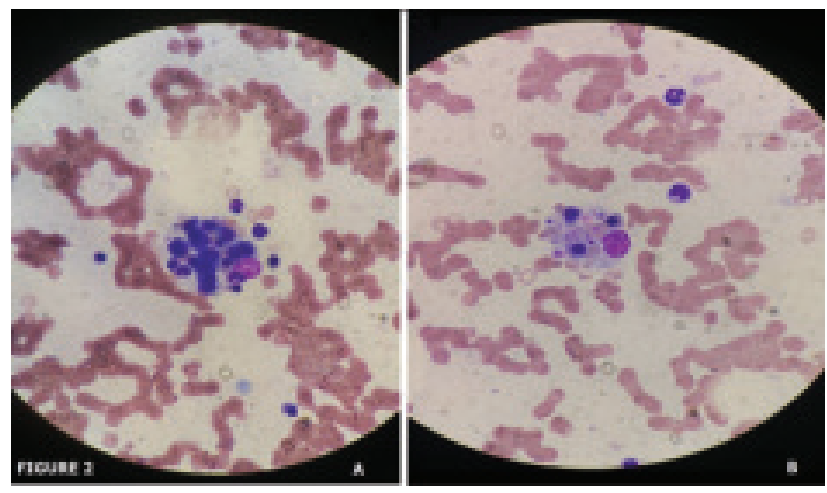

Figure 2. Histopathological Examination of bone marrow aspirate under May-Grunwald Giemsa stain shows increased number of histiocytes and prominent hemophagocytic activity which ingesting all three hemopoietic lineages.

A: Histiocytes ingesting erythroid cells and granulocytes (band neutrophils). B: Histiocytes ingesting platelets.

IV immunoglobulin G was given at $0.4 \mathrm{mg} / \mathrm{kg}$ daily for five days. IV methylprednisolone was continued for three days and then was changed to oral prednisolone $30 \mathrm{mg}$ once daily for another two days. Following the completion of IV immunoglobulin G, she showed marked improvement clinically and biochemically. She was stable enough to be transferred out of intensive care unit into general medical ward for continuity of care.

\section{Discussion}

Hemophagocytic Lymphohistiocytosis (HLH) is a rare hyperinflammatory condition characterized by excessive activation of macrophages and lymphocytes that leads to extensive multi-lineage cellular destruction. The exact mechanism of HLH is still remains unclear, but it is believed that the hallmark of HLH pathophysiology is the presence of high amount of proinflammatory cytokines that cause inappropriate proliferation of activated T-cells which stimulates macrophages in bone marrow that leads to phagocytosis of various lineage of blood cells ${ }^{1,2}$.

The clinical presentation of HLH is variable and highly nonspecific that can cause major diagnostic difficulties 
and led to therapeutic delay. In 2004, HLH-study group proposed a diagnostic criterion for $\mathrm{HLH}$, which consist of persistent fever, splenomegaly, bi-cytopenia, hypertriglyceridemia and/or hypofibrinogenemia, hemophagocytosis, low or absent natural killer cell activity, hyperferritinemia, and high soluble interleukin-2 receptor levels. ${ }^{3}$ One has to fulfil at least five out of eight of the criteria. Our patient has persistent fever, bi-cytopenia, elevated triglycerides, hypofibrinogenemia, hyperferritinemia and hyperphagocytosis as evidence from the histopathological examination of the bone marrow biopsy, which made her fulfilled the criteria. HLH can be broadly classified into two types, which are primary HLH and secondary HLH. Primary or familial HLH is caused by abnormality of the genes involved in the cytolytic secretory pathway and has autosomal recessive mode of inheritance. Secondary or reactive HLH has a much less understood pathophysiology and usually triggered by infections. But it can also trigger by malignancy such as T-cell lymphomas, and autoimmune disease.

Viral infections that are commonly linked to HLH are Epstein-Barr virus infection, cytomegalovirus infection, adenovirus infection, and viral hepatitis. However, in the recent years HLH has been increasingly recognised to occur in the background of Dengue infection. Dengue infection is caused by a virus that belongs to the Flaviviridae family, and are transmitted by Aedes mosquito. It is endemic in the urban area due to the abundancy of stagnated water which acts as the breeding site for the Aedes mosquito. The spectrum of presentation can range from asymptomatic infection to severe dengue in the form dengue haemorrhagic fever and dengue shock syndrome. ${ }^{4}$ The febrile phase usually last for seven days, with the defervescence phase typically occur after the third day. If the fever lasted more than seven days or recur during defervescence phase, secondary bacterial infection must be excluded or the possibility of secondary HLH should be suspected, like the patient in this case report.

The aim of management for infection-induced HLH is to treat the underlying infection. There are a few documented literatures that reported cases that recovered spontaneously with just supportive treatment ${ }^{5,6}$. However, high dose of corticosteroids is often required to suppress the hyperinflammatory state. Intravenous immunoglobulin G (IVIG) have been reported to be very effective in treating Dengue associated $\mathrm{HLH}^{6}$ and often used alone or in combination with high dose corticosteroids, as how we treated our patient successfully.

Although there are very limited cases reported on HLH that occur in the background of Dengue infection, but current evidence shown that the association between the virus and HLH are deemed to be very significant. ${ }^{7}$ And as the prevalence of Dengue is high in this region, it is expected that the number of Dengue-associated $\mathrm{HLH}$ cases are also high. But it is likely that the diagnosis is missed as a result of low suspicion index among the treating physicians. It is imperative to consider HLH as one of the possible differential diagnosis in prolonged fever in Dengue infected patient, as early recognition and prompt initiation of treatment is vital in management of HLH.

\section{Conclusion}

In conclusion, Dengue-associated Hemophagocytic Lymphohistiocytosis (HLH) is a rare sequalae of a very common viral infection. Although it is very fatal but it is potentially treatable if the appropriate treatments are given on time. Therefore, high level of suspicion is important in order to recognise this complication early. The purpose of this case report is to increase awareness about the condition and hopefully able to prevent HLHrelated death.

\section{References}

1. Imashuku S, Ueda I, Teramura T, et al. Occurrence of haemophagocytic lymphohistiocytosis at less than 1 year of age: analysis of 96 patients. Eur J Pediatr 2005;164:315-9. https://doi.org/10.1007/s00431005-1636-9. PMid:15731905

2. Karras A, Hermine O. Macrophage activation syndrome. Rev Med Interne. 2002;23:768-78. [PubMed: 12378830 ].https: / / doi.org/10.1016/S02488663(02)00673-2

3. Hemophagocytic Lymphohistiocytosis Study Group. Histiocyte Society. Treatment protocol of the second international HLH study 2004 (HLH-2004). Adapted from Henter JI, Elinder G, Ost A and the FHL Study Group of the Histiocyte Society. Diagnostic Guidelines for the Hemophagocytic Lymphohistiocytosis. Semin. Oncol. 18 (1991) 29-33.

4. Ghosh A, Dar L. Dengue vaccines: challenges, development, current status and prospects. Indian $\mathrm{J}$ Med Microbiol 2015;33:3-15.https://doi.org/10.4103/ 0255 0857.148369. PMid:25559995

5. Raju S, Kalyanaraman S, Swaminathan K, Nisha A, Praisid S. Hemophagocytic lymphohistiocytosis syndrome in Dengue hemorrhagic fever. Indian $\mathrm{J}$ Pediatr 2014;81(12):1381-3. https:/ / doi.org/10.1007/ s12098-014-1425-4. PMid:24728701

6. Wan Jamaludin WF, Periyasamy P, Wan Mat R, Abdul Wahid SF. Dengue infection associated hemophagocytic syndrome: therapeutic interventions and outcome. J Clin Virol 2015;69:91-5. https://doi.org/10.1016/ j.jcv.2015.06.004.PMid:26209387

7. Ray S, Kundu S, Saha M, Chakrabarti P. Hemophagocytic syndrome in classic dengue fever. Journal of global infectious diseases. 2011 Oct;3(4):399.https://doi.org/10.4103/0974777X.91068. PMid:22224008 PMCid:PMC3250000. 